Nature. 2008 August 14; 454(7206): 846-851. doi:10.1038/nature07181.

\title{
UCP2 mediates ghrelin's action on NPY/AgRP neurons by lowering free radicals
}

\author{
Zane B. Andrews ${ }^{1,2}$, Zhong-Wu Liü ${ }^{2,5}$, Nicholas Walllingford ${ }^{2}$, Derek M. Erion ${ }^{2}$, Erzsebet \\ Borok $^{2}$, Jeffery M. Friedman ${ }^{6}$, Matthias H. Tschöp ${ }^{7}$, Marya Shanabrough ${ }^{2}$, Gary Cline ${ }^{3}$, \\ Gerald I. Shulman ${ }^{3}$, Anna Coppola ${ }^{2}$, Xiao-Bing Gao ${ }^{2}$, Tamas L. Horvath ${ }^{1,2,4}$, and Sabrina \\ Diano $^{2,4}$ \\ ${ }^{1}$ Department of Section of Comparative Medicine, Yale University School of Medicine, New \\ Haven, Connecticut 06520, USA. \\ ${ }^{2}$ Department of Obstetrics, Gynecology \& Reproductive Sciences, Yale University School of \\ Medicine, New Haven, Connecticut 06520, USA. \\ ${ }^{3}$ Internal Medicine, Howard Hughes Medical Institute, Yale University School of Medicine, New \\ Haven, Connecticut 06520, USA. \\ ${ }^{4}$ Neurobiology, Yale University School of Medicine, New Haven, Connecticut 06520, USA. \\ ${ }^{5}$ Department of Neurobiology, Yunyang Medical College, Shiyan, Hubei 442000, China. \\ ${ }^{6}$ Laboratory of Molecular Genetics, Howard Hughes Medical Institute, Rockefeller University, New \\ York, New York 10021, USA. \\ ${ }^{7}$ Department of Psychiatry, University of Cincinnati, Cincinnati, Ohio 45237, USA.
}

\section{Abstract}

The gut-derived hormone ghrelin exerts its effect on the brain by regulating neuronal activity. Ghrelin-induced feeding behaviour is controlled by arcuate nucleus neurons that co-express neuropeptide Y and agouti-related protein (NPY/AgRP neurons). However, the intracellular mechanisms triggered by ghrelin to alter NPY/AgRP neuronal activity are poorly understood. Here we show that ghrelin initiates robust changes in hypothalamic mitochondrial respiration in mice that are dependent on uncoupling protein 2 (UCP2). Activation of this mitochondrial mechanism is critical for ghrelin-induced mitochondrial proliferation and electric activation of NPY/AgRP neurons, for ghrelin-triggered synaptic plasticity of pro-opiomelanocortin-expressing neurons, and for ghrelin-induced food intake. The UCP2-dependent action of ghrelin on NPY/ AgRP neurons is driven by a hypothalamic fatty acid oxidation pathway involving AMPK, CPT1

\footnotetext{
Author Information Correspondence and requests for materials should be addressed to T.L.H. (tamas.horvath@yale.edu) and S.D. (sabrina.diano@yale.edu)..

Author Contributions Z.B.A., S.D. and T.L.H. designed, executed and performed analysis of experiments and wrote the paper N.W., D.M.E. and A.C. contributed to the execution of the experiments. M.S. and E.B. contributed to the execution of electron microscopy experiments and analysis of the electron microscopic data. [Author: Revised sentence ok?Yes.] Z.-W.L. carried out electrophysiological recordings. X.-B.G. supervised and analysed the electrophysiological experiments. G.C. and G.I.S. designed, performed and analysed the LCFA CoA and NEFA measurements. J.M.F. and M.H.T. provided critical models and reagents for the study and contributed to the data analyses and discussions.
}

Supplementary Information is linked to the online version of the paper at www.nature.com/nature. 
and free radicals that are scavenged by UCP2. These results reveal a signalling modality connecting mitochondria-mediated effects of G-protein-coupled receptors on neuronal function and associated behaviour.

Ghrelin alters neuronal activity in various brain regions including the hypothalamus ${ }^{1}$, hippocampus ${ }^{2}$ and midbrain $^{3}$. Ghrelin activates NPY/AgRP neurons of the arcuate nucleus ${ }^{1,4}$ to suppress proopiomelanocortin (POMC) neuronal activity while promoting food intake. However, the intracellular mechanisms that mediate NPY/AgRP neuronal activation in response to ghrelin remain unknown.

Uncoupling proteins are inner-membrane mitochondrial proteins that are important for bioenergetics of diverse tissues ${ }^{5-8}$. UCP2 is strongly expressed in the hypothalamus including NPY/AgRP neurons of the arcuate nucleus ${ }^{9,10}$ where ghrelin receptors (growth hormone secretagogue receptor 1; GHSR) are also expressed ${ }^{11}$. Ghrelin enhances Ucp2 mRNA [Author: Note that we italicise at the gene and mRNA level (the gene symbol here follows that for mouse). Please check that this is consistent throughout the paper.] expression in the pancreas ${ }^{12}$, $\operatorname{liver}^{13}$ and white adipose tissue ${ }^{14}$, and hypothalamic Ucp2 mRNA levels and protein activity [Author: 'Ucp2 mRNA levels and protein activity' correct?Yes!] are elevated during fasting ${ }^{9}$, when circulating ghrelin is increased ${ }^{15}$. Thus, we analysed whether UCP2 is involved in hypothalamic neuronal responses to ghrelin and associated food intake.

\section{Ghrelin induces mitochondrial respiration via UCP2}

Immunohistochemistry revealed the presence of GHSR [Author: GHSR (singular) correct yes, $U c p 2^{-/-}$mice correct?] in greater than $90 \%$ of UCP2-expressing, $\beta$-galactosidasepositive arcuate nucleus neurons in $U c p 2^{-/-}$(ref. 16) mice (Supplementary Fig. 1a-c).

To analyse whether ghrelin affects mitochondrial respiration, we studied isolated hypothalamic synaptosomes ${ }^{17}$ in ghrelin- and saline-treated animals. Mitochondrial respiration (given as nmol of oxygen consumed per minute per $\mathrm{mg}$ protein) was significantly increased after adding pyruvate and malate (state 2; saline $32.07 \pm 1.38$ and ghrelin $44.83 \pm$ 3.15, $P<0.05$, wild-type mice). [Author: Revised sentence ok?Yes.] The subsequent addition of ADP (state 3 ) increased respiration in the ghrelin-treated mice relative to saline controls (saline $99.39 \pm 3.51$ and ghrelin $155.98 \pm 5.36$, [Author: 155.98 \pm ] $P<0.05$, wildtype mice). Ghrelin increased basal uncoupling status after ATP synthase inhibition with oligomycin (saline 27.2 \pm 2.2 and ghrelin $37.3 \pm 1.6, P<0.05$, wild-type mice; Fig. 1a) and free-fatty-acid (palmitate)-induced respiration (saline 150.0 \pm 5.7 and ghrelin $170.1 \pm 4.7, P$ $<0.05$, wild-type mice; Fig. 1b). Finally, total respiratory capacity induced by carbonylcyanide-4-(trifluoromethoxy)-phenylhydrazone (FCCP) was also increased by ghrelin (saline $297.0 \pm 7.7$ and ghrelin $427.9 \pm 19.2, P<0.05$, wild-type mice; Fig. $1 \mathrm{c}$ ). These increases were dependent on UCP2, as no significant elevation was seen for the above respiration parameters in $U c p 2^{-/-}$mice compared to their saline controls (Fig. 1a-c). Genipin, a compound reported to be a specific UCP2 inhibitor ${ }^{18}$, inhibited oxygen consumption in all states of mitochondrial respiration in all tissues studied from both wildtype and $U c p 2^{-/-}$mice (Supplementary Table 1). 
In assessing whether ghrelin's effect on UCP2 activation involves Ucp2 gene expression, we found that ghrelin produced a robust increase in hypothalamic Ucp 2 mRNA in wild-type mice, but not in $\mathrm{Gshr}^{-1-}$ mice (Supplementary Fig. 1d). Ghrelin also stimulated Npy gene expression in the arcuate nucleus in a UCP2- (Fig. 1e) and GHSR-dependent (Supplementary Fig. 1e) manner. Finally, ghrelin-induced AgRP expression also depended on UCP2 (Supplementary Fig. 1f). Ghrelin had no effect on POMC (Supplementary Fig. $1 \mathrm{~g})$.

\section{Ghrelin induces UCP2-mediated mitochondrial proliferation}

During fasting the number of mitochondria in NPY/AgRP perikarya increases in a UCP2dependent manner ${ }^{9}$. Ghrelin, which is elevated during fasting, also increased mitochondrial number in NPY neuronal perikarya in wild-type but not $U c p 2^{-/-}$mice (Fig. 1f and Supplementary Fig. 1h, i). The number of mitochondria in POMC-GFP perikarya was unaffected (Supplementary Fig. 1j). Because we observed a ghrelin-induced, UCP2- and GSHR-dependent increase in nuclear respiratory factor 1 (Nrfl; Fig. 1g) expression, [Author: $N r f 1$ correct, as this panel shows $N r f 1$ mRNA?Yes.] a transcription factor that stimulates mitochondrial biogenesis, we propose that the change in mitochondrial number in NPY/AgRP neurons may also involve de novo mitochondrial biogenesis. This is supported by the observation that peroxisome proliferator-activated receptor- $\gamma$ coactivator $1 \mathrm{a}$ (PGC1A) enhances mitochondrial biogenesis and respiration in association with robust induction of UCP2 and NRF1 (ref. 8).

The above results on perikaryal mitochondria number and from counts of synaptosomal mitochondria from hypothalamic tissue of wild-type or $U c p 2^{-1-}$ mice treated with ghrelin or saline (see Supplementary Fig. 2a-g), together with ghrelin's effect on state 3 respiration and total respiration capacity of synaptosomal mitochondria, suggest that elevated oxygen consumption by mitochondria in response to ghrelin arises in part from increased mitochondrial number. [Author: Revised sentence ok?Yes.]

\section{Ghrelin alters mitochondrial membrane potential via UCP2}

In peripheral tissues ${ }^{19,20} \mathrm{UCP} 2$ alters mitochondrial membrane potential. Thus, we analysed the mitochondrial membrane potential in synaptosomes of ghrelin- or saline-treated wildtype and $U c p 2^{-1-}$ mice. Ghrelin-treated wild-type synaptosomes exhibited lower mitochondrial membrane potential after addition of pyruvate and malate (state 2: $78.43 \pm$ $8.61 \%$ of saline values; $P<0.05$ ) and oligomycin (state $4: 75.13 \pm 4.87 \%$ of saline values; $P$ $<0.05$ ) compared to saline-treated wild-type synaptosomes (Fig. 1h). [Author: Revised sentence ok?Yes.] These differences were absent in $U c p 2^{-/-}$mice (Supplementary Fig. 3a). These observations suggest that a component of the altered oxygen consumption induced by ghrelin may also arise from increased uncoupled respiration; however, further studies are needed to strengthen these findings and study the basal differences in mitochondrial membrane potential kinetics in wild-type and $U c p 2^{-/-}$mice. 


\section{Ghrelin-induced NPY/AgRP activation is dependent on UCP2}

As a surrogate of neuronal activation, c-fos expression was examined in response to ghrelin in NPY neurons using mice expressing NPY-GFP on the $U c p 2^{-/-}$background (NPY-GFP/ $U c p 2^{-/-}$mice). [Author: Revised sentence ok?Yes.] After ghrelin injection, total NPY/cfos dual labelling was significantly attenuated in NPY-GFP/Ucp $2^{-/-}$mice compared to NPY-GFP/wild-type mice (wild type 602.33 $\pm 98.50, U c p 2^{-/-} 298.40 \pm 64.59, P<0.05$;

Fig. 2a and Supplementary Fig. 3b-d). Neither total NPY cell number nor arcuate nucleus volume was affected by genotype or ghrelin treatment (Supplementary Fig. 3e, f). No difference was observed in POMC-GFP/Ucp $2^{-/-}$mice (Supplementary Fig. $3 \mathrm{~g}-\mathrm{i}$ ).

Next, patch-clamp whole-cell electrophysiological recordings in slice preparations from NPY-GFP/wild type and NPY-GFP/Ucp $2^{-/-}$mice showed that ghrelin increased action potential firing in all NPY neurons; however, it was severely attenuated in $U c p 2^{-{ }^{--}}$mice compared to wild-type animals (Fig. $2 \mathrm{~b}$ and Supplementary Fig. 4a). This defect was specific to the action of ghrelin on NPY neurons, as glutamate produced normal responses in wild-type and $U c p 2^{--}$mice (Supplementary Fig. 4b). Ghrelin had no effect on POMC-GFP neuronal action potential frequency. These results are concordant with anatomical observations showing that $94 \%$ of NPY and just $8 \%$ of POMC neurons express GHSRs ${ }^{11}$.

\section{Ghrelin-induced synaptic plasticity is mediated by UCP2}

Ghrelin indirectly hyperpolarizes POMC neurons by activating inhibitory NPY/AgRP inputs ${ }^{1}$, and, consistent with this, ghrelin-induced recruitment of miniature inhibitory postsynaptic currents (mIPSCs) on POMC cells was significantly elevated in wild-type animals $(153.8 \pm 22.4 \%, n=5)$, but not in $U c p 2^{-/-}$mice $(100.5 \pm 2.5 \%, n=5$; Fig. $2 \mathrm{c}$ and Supplementary Fig. 4c).

Changes in mIPSCs are associated with synaptic plasticity ${ }^{21-23}$. Corresponding to the mIPSC measurements, ghrelin treatment increased symmetric, putatively inhibitory synapses on POMC neurons in wild-type (saline $8.33 \pm 0.97$ and ghrelin $16.55 \pm 1.68$ ) but not in $U c p 2^{-/-}$mice (saline $7.23 \pm 1.63$ and ghrelin $9.12 \pm 1.63$; Fig. 2d). Conversely, ghrelin significantly reduced the number of asymmetric, putatively excitatory synapses on POMC neurons in wild-type mice (saline $15.75 \pm 1.89$ and ghrelin $8.66 \pm 1.05$ ) but not in $U c p 2^{-/-}$mice (Supplementary Fig. 4d). Although the inhibitory input of POMC neurons arises partially from arcuate nucleus NPY/AgRP neurons, the origin of the stimulatory synapses needs further exploration.

\section{UCP2 mediates ghrelin-induced food intake}

To determine whether the UCP2-dependent cellular responses described above are relevant for behaviour, we analysed the feeding behaviour of wild-type and $U c p 2^{-{ }^{-}}$mice in response to ghrelin administration (intraperitoneal, $10 \mathrm{nmol}$ ) at the beginning of the dark phase (Fig. 2e). At 1, 2 and $3 \mathrm{~h}$ after injection, ghrelin-induced hyperphagia was severely attenuated in $U c p 2^{-I-}$ mice compared to wild-type mice ( $1 \mathrm{~h}$ : wild type $4.45 \pm 0.24, \mathrm{Ucp} 2^{-I-} 2.23 \pm 0.19$, $P<0.01 ; 2$ h: wild type $4.41 \pm 0.36, U c p 2^{-/-} 1.72 \pm 0.03, P<0.001$; Fig. 2 e, units are fold intake relative to saline controls). [Author: Revised sentence ok?Yes.] We and others 
recently demonstrated that ghrelin can act at multiple sites in the brain to induce food intake $\mathrm{e}^{3,21}$. Thus, we injected ghrelin $\left(500 \mathrm{pmol}^{-1} \mathrm{l}^{-1}\right)$ locally into the mediobasal hypothalamus or ventral tegmental area (VTA) (Supplementary Fig. 4e, f) [Author: Please define VTA. Done.] immediately before the dark phase. At all time points, intra-mediobasal hypothalamus ghrelin-induced food intake was significantly lower in $U c p 2^{-l-}$ mice compared to wild type (Fig. 2f). Intra-VTA, ghrelin-induced food intake was also attenuated in $U c p 2^{-{ }^{-}}$mice compared to wild type at $1 \mathrm{~h}$ but not at 2 or $3 \mathrm{~h}$ after ghrelin injection (Supplementary Fig. 4g). These data suggest that UCP2 regulates ghrelin-induced feeding behaviour in both the mediobasal hypothalamus and VTA. The longer lasting effect of ghrelin in the mediobasal hypothalamus suggests that mediobasal hypothalamus control dominates ghrelin-induced feeding behaviour and is consistent with the finding that mice with conditional knockout of arcuate nucleus NPY/AgRP neurons in adulthood do not respond to ghrelin ${ }^{24}$.

We also tested the effect of leptin-reduced food intake in $U c p 2^{-/-}$mice compared to wildtype controls. No significant effects were observed between the two groups (Supplementary Fig. 5a, b).

\section{Ghrelin induces AMPK and ACC phosphorylation}

Ghrelin increases AMPK activity within the hypothalamus ${ }^{25}$, including NPY/AgRP neurons ${ }^{26}$, which promotes feeding 25,27 and enhances mitochondrial biogenesis and function in the periphery ${ }^{28,29}$. We found that ghrelin promotes AMPK phosphorylation (pAMPK/ AMPK ratio) in a GHSR-dependent manner (Fig. 3a) in both wild-type and $U c p 2^{-/-}$mice (Fig. 3b), indicating that ghrelin's action on AMPK is downstream of GHSR but upstream of UCP2. We also observed that ghrelin elevates phosphorylated acetyl-CoA carboxylase (ACC), consistent with increased AMPK activity (Fig. 3b). [Author: Please define ACC. Done. Correct definition of i.c.v.?] Intracerebroventricular (i.c.v.) injection of a stimulator of AMPK activity, 5-aminoimidazole-4-carboxamide-1- $\beta$-D-ribofuranoside (AICAR), like ghrelin, increased both mitochondrial coupled and uncoupled respiration in the hypothalamus of wild-type but not $U c p 2^{-{ }^{--}}$mice (Supplementary Fig. 6a). In addition, AICAR promoted food intake in both wild-type and $\mathrm{GhSr}^{-1-}$ mice in a similar manner (Fig. $3 \mathrm{c}$ ), whereas AICAR-induced food intake was significantly diminished in $U c \mathrm{cp} 2^{-{ }_{-}^{-}}$mice compared to wild type (Fig. 3d). Intracerebroventricular injection [Author: Please clarify term 'central injection'] of compound C, an inhibitor of AMPK, inhibited food intake induced after peripheral ghrelin injections in wild-type mice, but did not further decrease food intake in $U c p 2^{-{ }_{-}}$animals (Fig. 3e), and, it also prevented ghrelin-induced elevations in Ucp2 mRNA levels in wild-type mice (Fig. 3f). [Author: Revised sentence ok?Yes.] These results confirm that ghrelin activates AMPK signalling through the GHSR, and reveal that AMPK requires UCP2 to exert an effect on feeding.

\section{Ghrelin-induced food intake is mediated by CPT1}

AMPK activation and ACC inhibition lead to a reduction in malonyl-CoA and release of carnitine palmitoyltransferase 1 (CPT1) from allosteric inhibition. Increased CPT1 activity enhances oxidation of long chain fatty acids (LCFA) and increases food intake ${ }^{30}$. Ghrelin 
increased the expression of hypothalamic Cpt1 mRNA (Fig. 3g) and this was dependent on AMPK activity, as intracerebroventricularly administered compound $\mathrm{C}$ blocked this effect (Fig. 3g). The induction of Cptl mRNA by ghrelin occurred rapidly, after just $1 \mathrm{~h}$ (Supplementary Fig. 6b). Ghrelin-triggered elevation in Ucp2, Npy and Nrf1 mRNA levels also occurred after $1 \mathrm{~h}$ (Supplementary Fig. 6c). The ghrelin-induced increase in Ucp2 mRNA levels was diminished by concomitant intracerebroventricular administration of either compound $\mathrm{C}$ or etomoxir (CPT1 inhibitor) (Fig. 3f), confirming that ghrelin-induced Ucp 2 mRNA expression is mediated by the AMPK-CPT1 pathway. Furthermore, etomoxir and compound $\mathrm{C}$ prevented the ghrelin-induced increase in both Npy and Agrp gene expression (Fig. 3h and Supplementary Fig. 6d), mimicking the impairments found in $U c p 2^{-l-}$ mice (see Fig. 1e and Supplementary Fig. 1f). No difference in Pomc mRNA was observed in any group (data not shown). In line with the mRNA expression data, etomoxir also suppressed ghrelin-induced food intake (Fig. 3i).

The aforementioned data indicate that CPT1 activation, similar to AMPK activation, is upstream of UCP2. However, we found that ghrelin-induced elevation of hypothalamic Cpt1 mRNA levels is not observable in $U c p 2^{-/-}$animals (Fig. $3 \mathrm{j}$ ). This apparent paradox is resolved in studies described below regarding hypothalamic free radicals.

\section{Ghrelin raises hypothalamic LCFA CoAs and blood NEFAs}

To assess whether ghrelin increases the availability of substrates for mitochondrial beta oxidation coinciding with AMPK and CPT1 activation, we analysed hypothalamic levels of LCFA CoAs $3 \mathrm{~h}$ after ghrelin administration. Total LCFA CoAs (comprising both 16- and 18-carbon LCFA CoAs) were significantly higher in the hypothalamus of ghrelin- versus saline-treated controls (saline $29.15 \pm 1.17$, ghrelin $33.72 \pm 1.15 \mathrm{nmol}$ metabolite per $\mathrm{g}$ tissue; $P<0.05 ; n=4$ and 6 respectively; Supplementary Fig. 6e). Next, we analysed LCFA CoAs in the hypothalamus at 0, 30, 60,120 and 180 min after ghrelin administration. Compared to time 0 ( $36.18 \pm 2.25 \mathrm{nmol}$ metabolite per $\mathrm{g}$ tissue), by $30 \mathrm{~min}$ (in the absence of food) hypothalamic LCFA CoA levels were significantly elevated $(40.75 \pm 1.55 \mathrm{nmol}$ metabolite per $\mathrm{g}$ tissue, $P<0.05)$ and remained high through $60(43.08 \pm 0.95 \mathrm{nmol}$ metabolite per g tissue), 120 (42.24 $\pm 0.62 \mathrm{nmol}$ metabolite per g tissue) and $180 \mathrm{~min}$ (40.9 $\pm 0.42 \mathrm{nmol}$ metabolite per g tissue; Supplementary Fig. 6f). Although no statistical differences were detected between these values, the trend suggests that from $60 \mathrm{~min}$ onwards, hypothalamic levels of LCFA CoAs may start to descend (Supplementary Fig. 6f), perhaps due to increased use and oxidation of LCFA CoAs. Supporting this idea is the paradigm in which chronically elevated ghrelin ( $20 \mathrm{~h}$ of fasting) resulted in depletion of hypothalamic levels of LCFA CoAs ${ }^{31}$. We also found that non-esterified fatty acid (NEFA) levels in the circulation ( $0.23 \pm 0.3$ mequiv. $1^{-1}$ at time 0$)$ start to rise 30 min after ghrelin injection $\left(0.30 \pm 0.3\right.$ mequiv. $\left.1^{-1}, P>0.05\right)$, and become significantly higher at $60(0.42 \pm$ 0.3 mequiv. $\left.1^{-1}, P<0.01\right), 120\left(0.51 \pm 0.07\right.$ mequiv. $\left.1^{-1}\right)$ and 1,080 min [Author: Do you mean $180 \mathrm{~min}$ ? No, it is 1,080 min as 18 hours. mequiv. for milliequivalent ok? Yes.] (1.09 \pm 0.11 mequiv. $1^{-1}, P<0.00001$ ) (Supplementary Fig. $6 \mathrm{~g}$ ). The elevated NEFA levels during negative energy balance accompanied by an initial rise but subsequent decline in hypothalamic LCFA CoA levels is consistent with the conclusions of ref. 31 suggesting that 
the source of hypothalamic LCFA CoAs is the periphery, and also with our observations that hypothalamic LCFA CoAs may undergo beta oxidation.

\section{Ghrelin reduces ROS in NPY/AgRP neurons via UCP2}

A by-product of increased mitochondrial fatty acid beta oxidation is increased ROS production ${ }^{32,33}$. Ghrelin significantly increased synaptosomal ROS in $U c p 2^{-{ }_{-}^{-}}$but not in wild-type hypothalami compared to saline controls (Fig. 4a). Next, we injected ghrelin or saline together with dihydroethidium bromide (DHE), a compound that fluoresces as it is oxidized, to analyse in situ ROS levels in NPY-GFP and POMC-GFP mice on wild-type and $U c p 2^{-/-}$backgrounds. There was no difference in DHE levels [Author: Do you mean there was no difference in the amount of fluorescent DHE?Yes.] in NPY neurons of wildtype animals treated with either ghrelin or saline (Fig. 4b and Supplementary Fig. 7). However, ghrelin treatment of $U c p 2^{-/-}$animals resulted in increased levels of DHE in NPY neurons compared to saline controls (Fig. 4b and Supplementary Fig. 7). The analysis of randomly selected, non-GFP-positive cells showed a shift in DHE levels similar to that seen in NPY neurons (quantified data not shown). In contrast, however, DHE levels in POMCGFP neurons were significantly higher in wild-type mice treated with saline compared to those given ghrelin (Fig. $4 \mathrm{~b}$ and Supplementary Fig. 7). In $U c p 2^{-{ }^{-}}$mice, lower levels of DHE were observed in POMC neurons in both the saline- and ghrelin-treated mice (Fig. 4b and Supplementary Fig. 7). Overall these data are consistent with UCP2's putative ability to buffer ROS levels.

\section{ROS scavenging reverses responses of Ucp2-/- mice}

In light of the data on ROS, we hypothesized that the paradoxical lack of Cpt1 mRNA induction by ghrelin in $U c p 2^{--}$animals (Fig. 3j) may be due to an effect of ROS on CPT1. Thus, we injected a ROS scavenging cocktail containing ascorbic acid $(10 \mu \mathrm{M}), N$-acetyl-Lcysteine $(10 \mu \mathrm{M})$ and TEMPO $(1 \mu \mathrm{M})$ intracerebroventricularly into wild-type or $U c p 2^{-/-}$ mice with a concurrent intraperitoneal injection of ghrelin (10 nmol). Scavenging of ROS enabled ghrelin to induce elevated $C p t 1$ mRNA levels in $U c p 2^{-/-}$mice (Fig. 4c).

Our results support the hypothesis that ghrelin-triggered ROS production promotes UCP2 activity and mRNA expression and that the main action of UCP2 in these circuits is to promote ROS scavenging. In line with this, we found that after intracerebroventricular injection of ROS scavenging cocktail, ghrelin-induced elevations in hypothalamic Ucp2 mRNA levels in wild-type mice were diminished (Supplementary Fig. 8a), which was paralleled by rescue of induction of Npy and Agrp mRNA levels by ghrelin in Ucp $2^{-/-}$mice (Fig. 4d and Supplementary Fig. 8b). In addition, incubating isolated hypothalamic synaptosomes from ghrelin-treated wild-type mice with the ROS scavenging cocktail suppressed ghrelin-induced increases in mitochondrial uncoupled respiration after oligomycin treatment but not in state 3 or FCCP-driven respiration (Supplementary Fig. 8c). When hypothalamic slices from $U c p 2^{-/-}$mice were incubated (for $20 \mathrm{~min}$ ) with the ROS scavenging cocktail, ghrelin-evoked NPY neuronal firing was also restored to wild-type values (Figs $2 \mathrm{~b}$ and $4 \mathrm{e}$ ). Moreover, in the presence of the ROS scavenger, washout of ghrelin did not silence NPY neurons (Fig. 4e), indicating that the intracellular events 
triggered by ghrelin not only potentiated but also maintained neuronal firing subsequent to the initial ghrelin signalling. In association with the effect of ROS scavenger on cellular events in the hypothalamus of $U c p 2^{--}$mice, ghrelin's ability to promote feeding was restored as well in $U c p 2^{--}$mice pre-treated with the ROS scavenger (Fig. 4f).

\section{Discussion}

We have identified intracellular events that contribute to appropriate activation of NPY/ AgRP neurons in response to ghrelin (Supplementary Fig. 9). Ghrelin induces a rapid increase of NPY/AgRP neuronal firing by activation of GHSR. Ghrelin, via GHSR, also results in AMPK activation that suppresses ACC activity, eliminating the inhibition of CPT1. CPT1 activation can enhance LCFA entry into mitochondria and increase beta oxidation. Fatty acid beta oxidation promotes generation of $\operatorname{ROS}^{32,33}$, which together with fatty acids promote UCP2 transcription and activity ${ }^{34}$. UCP2 activity neutralizes ROS $^{35}$, thereby allowing continuous CPT1-promoted fatty acid beta oxidation that enables continuous support of the bioenergetic needs to maintain firing of NPY/AgRP cells. Sustained firing of NPY/AgRP neurons, the efferents of which are GABAergic ${ }^{36,37}$ onto POMC neurons, results in activity-dependent synaptic plasticity promoting an organization of increased inhibitory input onto POMC neurons leading to increased feeding. Our results also indicate that fatty acids, which are elevated in the circulation concurrently with ghrelin during fasting ${ }^{15}$, are acutely increased in the hypothalamus in response to ghrelin administration so that they can serve as a continuous fuel supply for NPY/AgRP neurons during negative energy balance. In light of previous work ${ }^{34}$, we predict that the absence of fatty acid beta oxidation such as during increased carbohydrate load or positive energy balance may affect UCP2 activation and lead to a different cellular outcome. In support of this, it was recently reported that elevated glucose levels in animals on a high fat diet promote UCP2 induction, which was paralleled with impaired ability of POMC neurons to sense glucose ${ }^{38}$. The apparent elevated baseline ROS levels in POMC neurons are consistent with the possibility of increased carbohydrate fuel-based activity in these cells at the time of relative satiety (when UCP2 would not be activated), because carbohydrates, and glucose in particular, are major generators of ROS through increasing electron transport at complex 1. Because NPY neurons show lower ROS production than POMC neurons, it is tempting to speculate that the NPY/AgRP neurons are less susceptible to free-radical-induced damage, leading to resistance of orexigenic hypothalamic mechanisms to cellular stress. Conversely, POMC cells may be more vulnerable to free-radical-induced damage and progressive degeneration, which may have relevance to age-related onset of obesity. Because GHSR and UCP2 expression overlaps in other brain sites, including the midbrain, we predict that ghrelin modulation of other brain functions will also be regulated by UCP2-modulated fatty acid metabolism.

\section{METHODS SUMMARY}

Detailed methodology can be found in the Supplementary Information. In short, tissue preparation, immunocytochemistry, synaptosomal respiration, Real-time PCR, quantitative and qualitative electron microscopic analyses of mitochondria and synapse number, 
electrophysiology and feeding behaviour analyses were performed as previously described $^{1-3,9,23,36,39}$.

The mitochondrial membrane potential was measured in isolated synaptosomal mitochondrial fractions from the hypothalamus using $\Delta \Psi_{\mathrm{m}}$-sensitive TMRE and fluorescent spectrofluorophotometry. Non-esterified fatty acids were measured in plasma using a colorimetric non-esterified fatty acid (NEFA) kit (Wako) following the manufacturer's instructions. LCFA CoAs were measured by mass spectroscopy.

ROS production was quantified using dichlorodihydrofluorescein diacetate (DCF) in mitochondrial fractions and using DHE in fixed tissue.

\section{Supplementary Material}

Refer to Web version on PubMed Central for supplementary material.

\section{Acknowledgments}

This work was supported by NIH grants to T.L.H., S.D, X.-B.G. and G.I.S., by a New Zealand Foundation for Research Science and Technology (FRST) fellowship to Z.B.A,by grants from the JDRF and American Diabetes Association to S.D, and by a grant from the Michael J. Fox Foundation to T.L.H. We thank B. Lowell for providing breeding pairs of $U c p 2^{-I-}$ mice and M. Sleeman for providing breeding pairs of $\mathrm{Ghsr}^{-1}$ mice. S.D. thanks A. Lombardi for the discussion on mitochondrial membrane potential measurements. The authors thank V. Pieribone for the use of a spectrofluorophotometer.

\section{References}

1. Cowley MA, et al. The distribution and mechanism of action of ghrelin in the CNS demonstrates a novel hypothalamic circuit regulating energy homeostasis. Neuron. 2003; 37:649-661. [PubMed: 12597862]

2. Diano $\mathrm{S}$, et al. Ghrelin controls hippocampal spine synapse density and memory performance. Nature Neurosci. 2006; 9:381-388. [PubMed: 16491079]

3. Abizaid A, et al. Ghrelin modulates the activity and synaptic input organization of midbrain dopamine neurons while promoting appetite. J. Clin. Invest. 2006; 116:3229-3239. [PubMed: 17060947]

4. Kamegai J, et al. Central effect of ghrelin, an endogenous growth hormone secretagogue, on hypothalamic peptide gene expression. Endocrinology. 2000; 141:4797-4800. [PubMed: 11108296]

5. Diano $S$, et al. Uncoupling protein 2 prevents neuronal death including that occurring during seizures: a mechanism for preconditioning. Endocrinology. 2003; 144:5014-5021. [PubMed: 12960023]

6. Garcia-Martinez C, et al. Overexpression of UCP3 in cultured human muscle lowers mitochondrial membrane potential, raises ATP/ADP ratio, and favors fatty acid vs. glucose oxidation. FASEB J. 2001; 15:2033-2035. [PubMed: 11511517]

7. Rossmeisl M, et al. Expression of the uncoupling protein 1 from the aP2 gene promoter stimulates mitochondrial biogenesis in unilocular adipocytes in vivo. Eur. J. Biochem. 2002; 269:19-28. [PubMed: 11784294]

8. Wu Z, et al. Mechanisms controlling mitochondrial biogenesis and respiration through the thermogenic coactivator PGC-1. Cell. 1999; 98:115-124. [PubMed: 10412986]

9. Coppola A, et al. A central thermogenic-like mechanism in feeding regulation: an interplay between arcuate nucleus T3 and UCP2. Cell Metab. 2007; 5:21-33. [PubMed: 17189204]

10. Horvath TL, et al. Brain uncoupling protein 2: uncoupled neuronal mitochondria predict thermal synapses in homeostatic centers. J. Neurosci. 1999; 19:10417-10427. [PubMed: 10575039] 
11. Willesen MG, Kristensen P, Romer J. Co-localization of growth hormone secretagogue receptor and NPY mRNA in the arcuate nucleus of the rat. Neuroendocrinology. 1999; 70:306-316. [PubMed: 10567856]

12. Sun Y, Asnicar M, Saha PK, Chan L, Smith RG. Ablation of ghrelin improves the diabetic but not obese phenotype of ob/ob mice. Cell Metab. 2006; 3:379-386. [PubMed: 16679295]

13. Barazzoni R, et al. Ghrelin regulates mitochondrial-lipid metabolism gene expression and tissue fat distribution in liver and skeletal muscle. Am. J. Physiol. Endocrinol. Metab. 2005; 288:E228E235. [PubMed: 15328073]

14. Tsubone T, et al. Ghrelin regulates adiposity in white adipose tissue and UCP1 mRNA expression in brown adipose tissue in mice. Regul. Pept. 2005; 130:97-103. [PubMed: 15946750]

15. Tschop M, Smiley DL, Heiman ML. Ghrelin induces adiposity in rodents. Nature. 2000; 407:908913. [PubMed: 11057670]

16. Horvath B, et al. Uncoupling protein 2 (UCP2) lowers alcohol sensitivity and pain threshold. Biochem. Pharmacol. 2002; 64:369-374. [PubMed: 12147287]

17. Scott ID, Nicholls DG. Energy transduction in intact synaptosomes. Influence of plasmamembrane depolarization on the respiration and membrane potential of internal mitochondria determined in situ. Biochem. J. 1980; 186:21-33. [PubMed: 7370008]

18. Zhang CY, et al. Genipin inhibits UCP2-mediated proton leak and acutely reverses obesity- and high glucose-induced beta cell dysfunction in isolated pancreatic islets. Cell Metab. 2006; 3:417427. [PubMed: 16753577]

19. Krauss S, Zhang CY, Lowell BB. A significant portion of mitochondrial proton leak in intact thymocytes depends on expression of UCP2. Proc. Natl Acad. Sci. USA. 2002; 99:118-122. [PubMed: 11756659]

20. Zhang CY, et al. Uncoupling protein-2 negatively regulates insulin secretion and is a major link between obesity, beta cell dysfunction, and type 2 diabetes. Cell. 2001; 105:745-755. [PubMed: 11440717]

21. Abizaid A, Gao Q, Horvath TL. Thoughts for food: brain mechanisms and peripheral energy balance. Neuron. 2006; 51:691-702. [PubMed: 16982416]

22. Horvath TL, Gao XB. Input organization and plasticity of hypocretin neurons: possible clues to obesity's association with insomnia. Cell Metab. 2005; 1:279-286. [PubMed: 16054072]

23. Pinto S, et al. Rapid rewiring of arcuate nucleus feeding circuits by leptin. Science. $2004 ; 304: 110$ 115. [PubMed: 15064421]

24. Luquet S, Phillips CT, Palmiter RD. NPY/AgRP neurons are not essential for feeding responses to glucoprivation. Peptides. 2007; 28:214-225. [PubMed: 17194499]

25. Andersson U, et al. AMP-activated protein kinase plays a role in the control of food intake. J. Biol. Chem. 2004; 279:12005-12008. [PubMed: 14742438]

26. Kohno D, Sone H, Minokoshi Y, Yada T. Ghrelin raises $[\mathrm{Ca} 2+]_{\mathrm{i}}$ via AMPK in hypothalamic arcuate nucleus NPY neurons. Biochem. Biophys. Res. Commun. 2008; 366:388-392. [PubMed: 18068666]

27. Minokoshi Y, et al. AMP-kinase regulates food intake by responding to hormonal and nutrient signals in the hypothalamus. Nature. 2004; 428:569-574. [PubMed: 15058305]

28. Bergeron R, et al. Chronic activation of AMP kinase results in NRF-1 activation and mitochondrial biogenesis. Am. J. Physiol. Endocrinol. Metab. 2001; 281:E1340-E1346. [PubMed: 11701451]

29. Reznick RM, Shulman GI. The role of AMP-activated protein kinase in mitochondrial biogenesis. J. Physiol. 2006; 574:33-39. [PubMed: 16709637]

30. Lam TK, Schwartz GJ, Rossetti L. Hypothalamic sensing of fatty acids. Nature Neurosci. 2005; 8:579-584. [PubMed: 15856066]

31. Wolfgang MJ, et al. Regulation of hypothalamic malonyl-CoA by central glucose and leptin. Proc. Natl Acad. Sci. USA. 2007; 104:19285-19290. [PubMed: 18032600]

32. Du X, et al. Insulin resistance reduces arterial prostacyclin synthase and eNOS activities by increasing endothelial fatty acid oxidation. J. Clin. Invest. 2006; 116:1071-1080. [PubMed: 16528409] 
33. Yamagishi SI, et al. Leptin induces mitochondrial superoxide production and monocyte chemoattractant protein-1 expression in aortic endothelial cells by increasing fatty acid oxidation via protein kinase A. J. Biol. Chem. 2001; 276:25096-25100. [PubMed: 11342529]

34. Echtay KS, et al. Superoxide activates mitochondrial uncoupling proteins. Nature. 2002; 415:9699. [PubMed: 11780125]

35. Brand MD, et al. Mitochondrial superoxide: production, biological effects, and activation of uncoupling proteins. Free Radic. Biol. Med. 2004; 37:755-767. [PubMed: 15304252]

36. Cowley MA, et al. Leptin activates anorexigenic POMC neurons through a neural network in the arcuate nucleus. Nature. 2001; 411:480-484. [PubMed: 11373681]

37. Horvath TL, Bechmann I, Naftolin F, Kalra SP, Leranth C. Heterogeneity in the neuropeptide Ycontaining neurons of the rat arcuate nucleus: GABAergic and non-GABAergic subpopulations. Brain Res. 1997; 756:283-286. [PubMed: 9187344]

38. Parton LE, et al. Glucose sensing by POMC neurons regulates glucose homeostasis and is impaired in obesity. Nature. 2007; 449:228-232. [PubMed: 17728716]

39. Gao Q, et al. Anorectic estrogen mimics leptin's effect on the rewiring of melanocortin cells and Stat3 signaling in obese animals. Nature Med. 2007; 13:89-94. [PubMed: 17195839] 

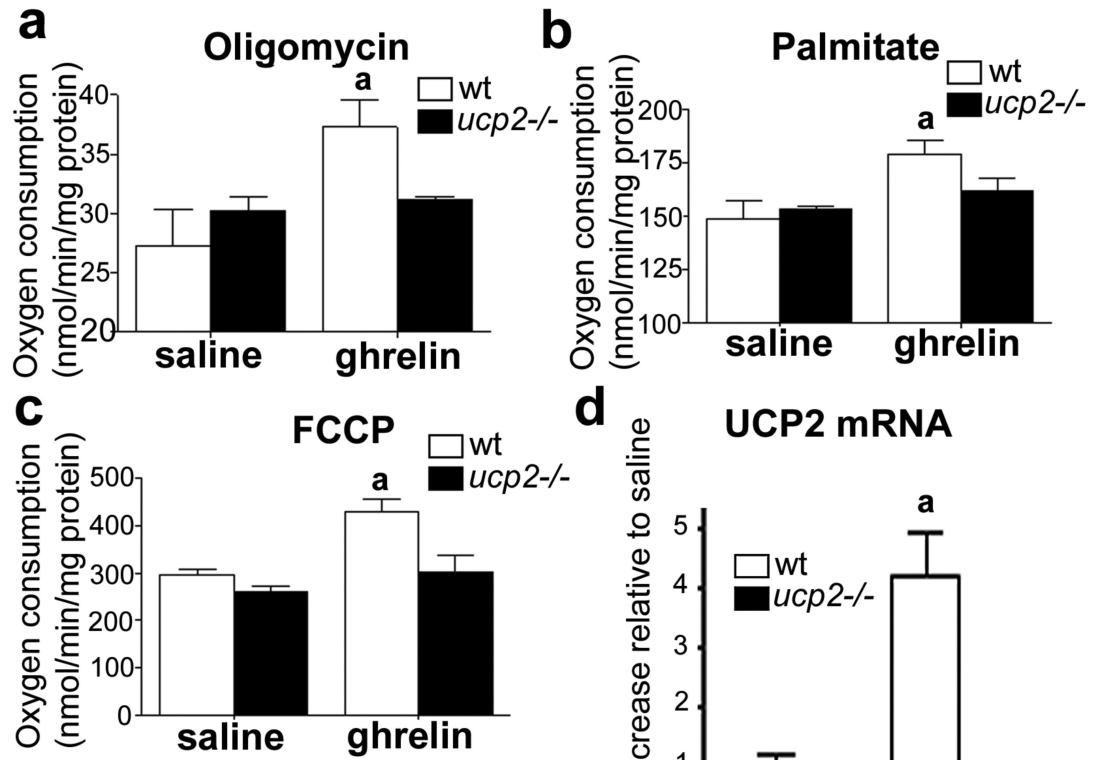

\section{d UCP2 mRNA}

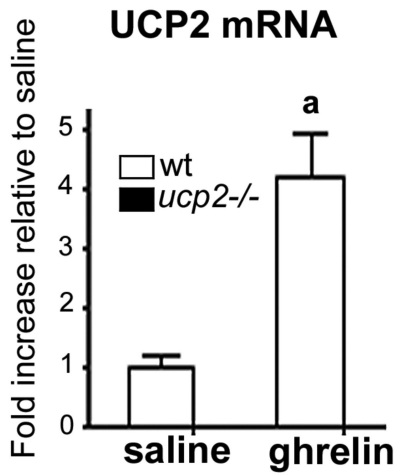

e
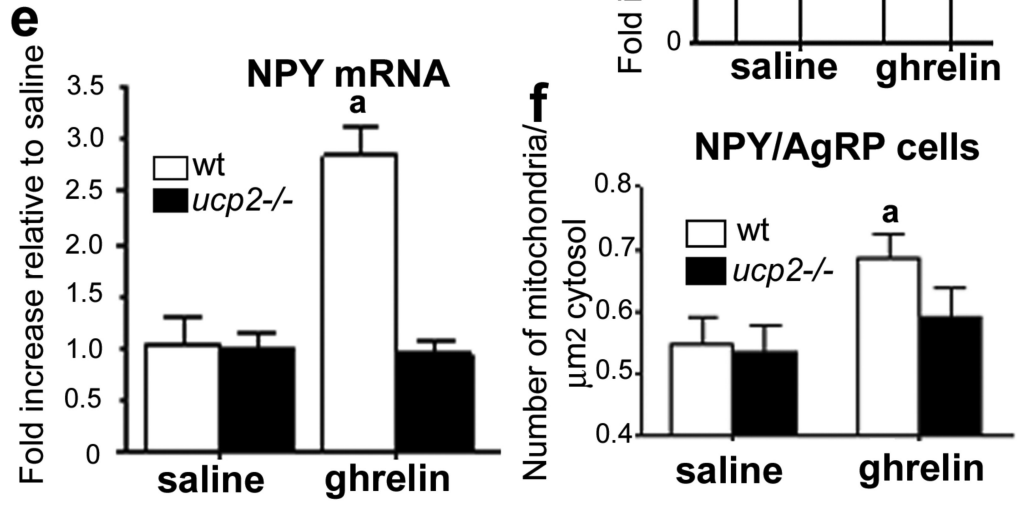

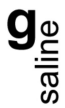

NRF1 mRNA
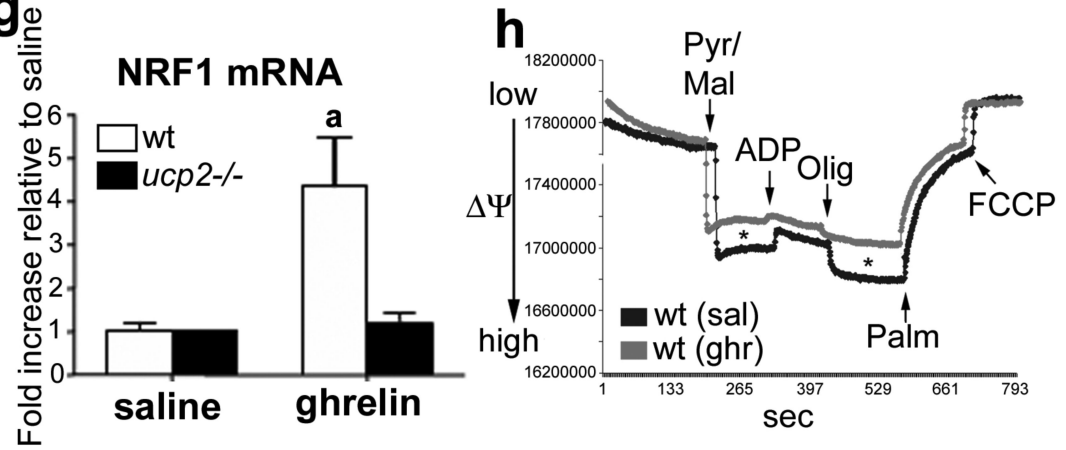

Figure 1. Mitochondrial and transcriptional effects of ghrelin are UCP2-mediated a-c, Ghrelin alters hypothalamic mitochondrial respiration. a, Oligomycin inhibits ATP synthase and uncouples substrate oxidation from ATP phosphorylation. The resulting increase in uncoupled oxygen consumption in ghrelin-treated mice is due to UCP2, as $U c p 2^{-1-}$ mice exhibit no response to ghrelin. b, Ghrelin enhances respiration after palmitate addition in wild-type but not $U c p 2^{-/-}$mice. [Author: Revised sentence ok?Yes.] c, An increase in respiration after FCCP shows that ghrelin enhances the maximal respiratory capacity in wild-type but not in $U c p 2^{-/-}$mice. d, Ghrelin increases $(10 \mathrm{nmol})$ hypothalamic 
Ucp 2 mRNA expression as measured by real-time PCR $(n=6)$. The data are expressed as a fold increase relative to saline levels. e, Arcuate $N p y$ gene expression is increased in response to ghrelin in wild-type but not $U c p 2^{-/-}$mice $(n=6)$. f, Ghrelin promotes mitochondrial proliferation in a UCP2-dependent manner in arcuate NPY/AgRP cells. [Author: Should this be NPY/AgRP cells, as per panel label?Yes.] g, Ghrelin increases Nrfl mRNA expression in the hypothalamus of wild-type but not $U c p 2^{-/-}$mice. $\mathbf{h}$, Representative traces showing that ghrelin decreases the mitochondrial membrane potential after addition of energy substrates (state 2) and after addition of oligomycin (state 4). Asterisk indicates statistically significant differences $(P<0.05)$ with respect to saline controls. All error bars indicate s.e.m. [Author: Correct definition of error bars?Yes.] Olig, oligomycin; Palm, palmitate; Pyr/Mal, pyruvate and malate. [Author: Correct definitions? $\Delta \Psi_{\mathbf{m}}$ correct for $\mathbf{y}$ axis label?Yes.] 

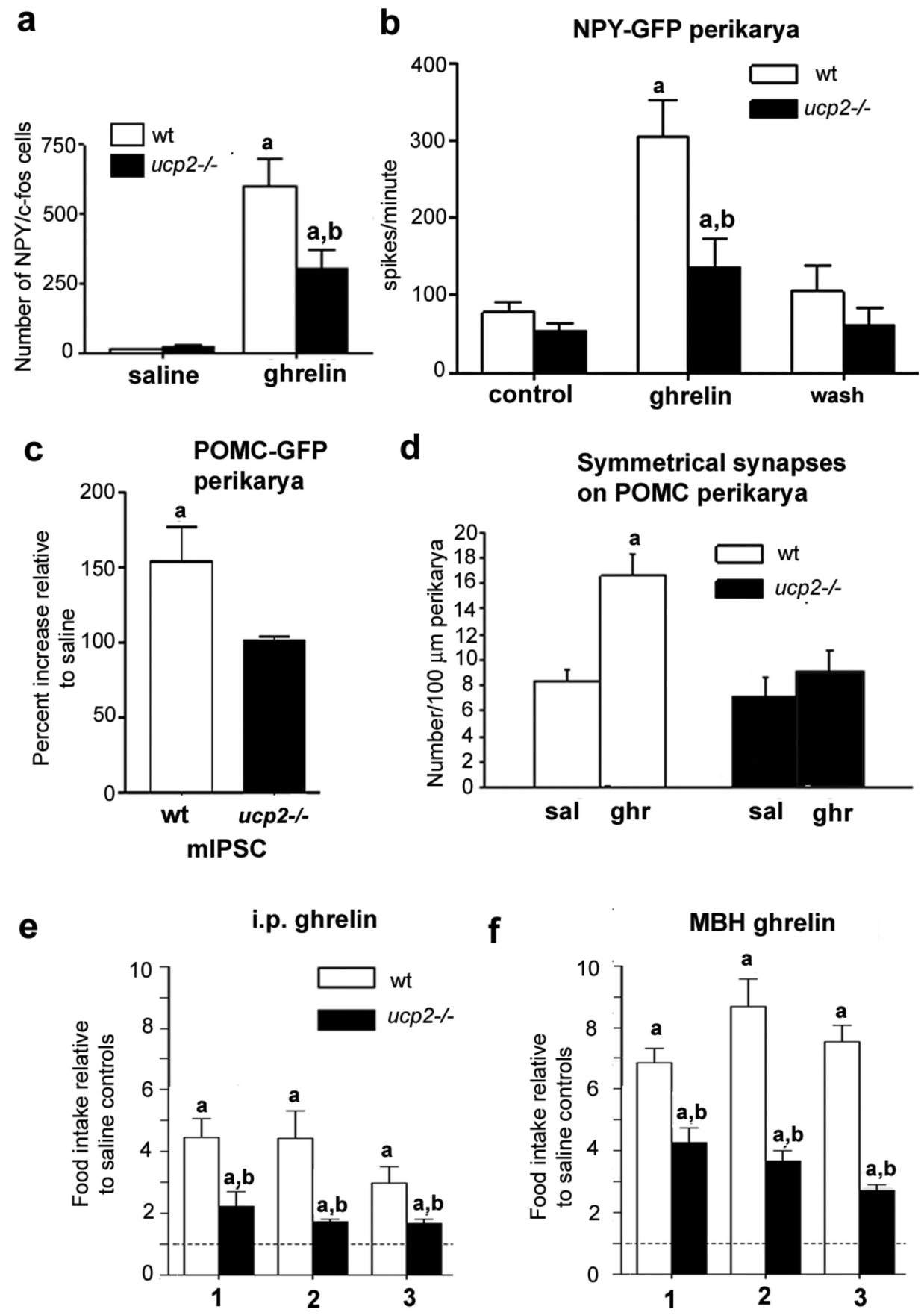

Figure 2. Effects of ghrelin on neuronal firing, synaptic plasticity and feeding are UCP2mediated

a, UCP2 potentiates NPY c-fos activation in the arcuate nucleus in response to ghrelin (10 $\mathrm{nmol})$. Stereological analysis of total NPY-GFP/c-fos cells in the arcuate nucleus of wildtype $(n=6)$ and $U c p 2^{-/-}$mice $(n=6)$ is shown. $\mathbf{b}$, Ghrelin increases mean action potential frequency of NPY-GFP neuronal perikarya in whole-cell recordings from wild-type mice $(n$ =7); this effect is diminished in $U c p 2^{-/-}$mice $(n=7)$. [Author: Revised sentence ok?] c, Ghrelin increases the frequency of mIPSCs on POMC-GFP perikarya in wild type but not in 
$U c p 2^{-/-}$mice ( $n=5$ for both groups). d, Ghrelin increases the number of symmetric, putatively inhibitory perikaryal synapses on POMC neurons in wild-type mice but not in $U c p 2^{-I-}$ mice. [Author: 'Number per $100 \mu \mathrm{m}$ [micrometres] perikarya' correct for y axis of d?Yes.] e, Peripheral ghrelin administration (intraperitoneal) significantly $(P<0.05)$ enhances food intake relative to saline controls in both wild-type $(n=6)$ and $U c p 2^{-{ }^{-}}(n=6)$ mice at 1,2 and $3 \mathrm{~h}$ after injection. However, food intake in $U c p 2^{-{ }^{--}}$mice is significantly attenuated compared to wild-type mice 1, 2 and $3 \mathrm{~h}$ after injection. $\mathbf{f}$, Mediobasal

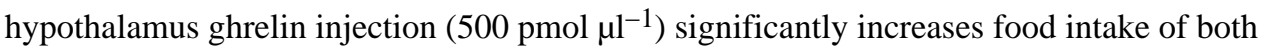
genotypes at 1,2 and $3 \mathrm{~h}$ after injection. However, $U c p 2^{-{ }^{-}}$mice display severely attenuated food intake at all time points compared to wild-type controls. Asterisk indicates statistically significant differences $(P<0.05)$ with respect to saline controls; dagger indicates statistically significant $(P<0.05)$ reduction with respect to ghrelin-treated wild-type mice. All error bars indicate s.e.m. [Author: Correct definition of error bars?Yes.] 

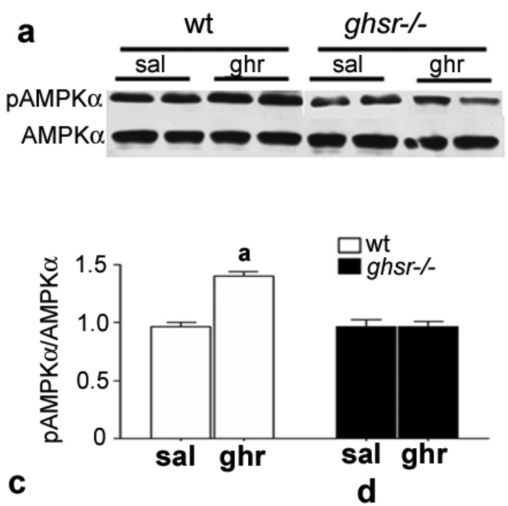

b
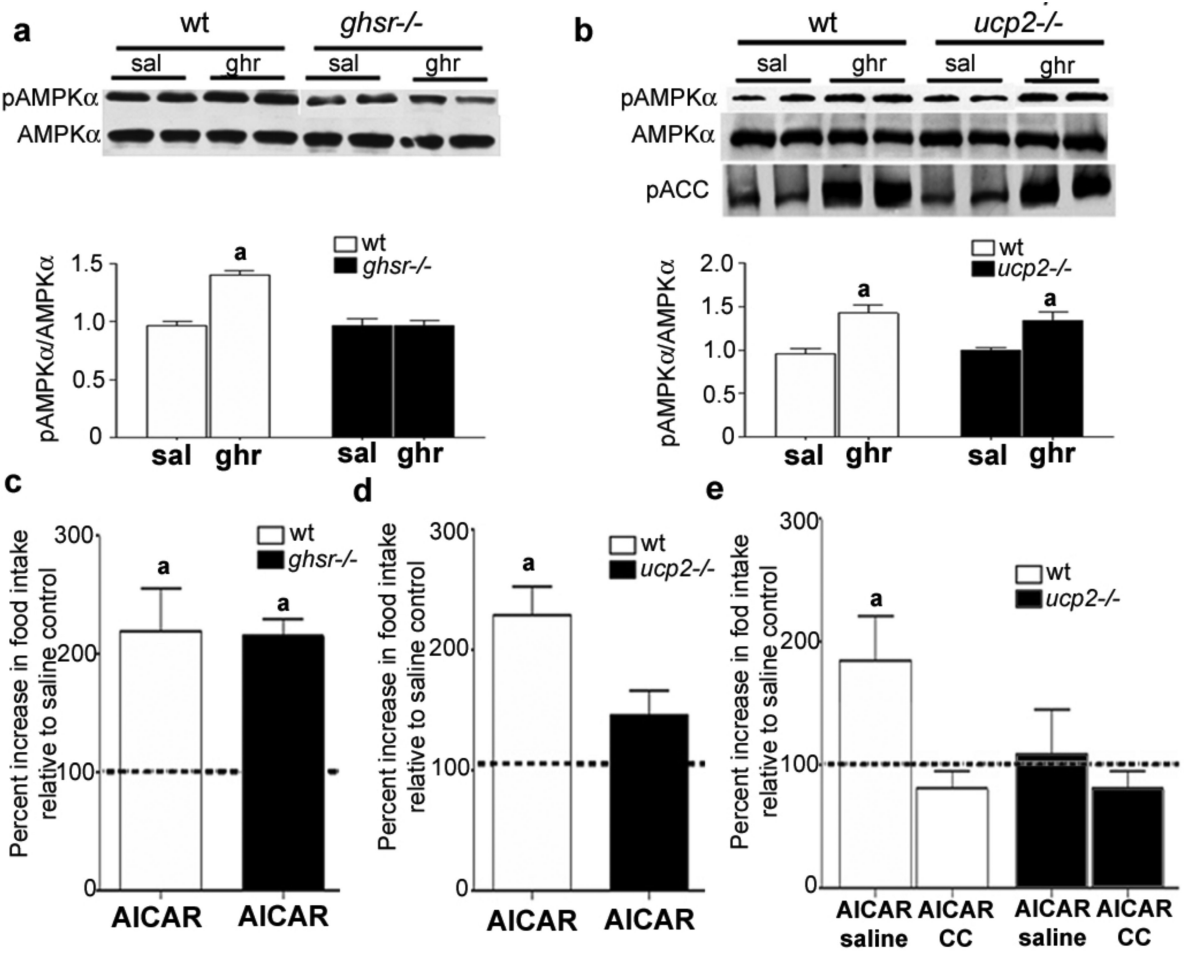

f

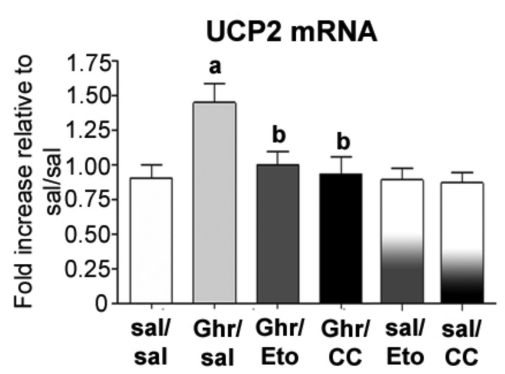

g
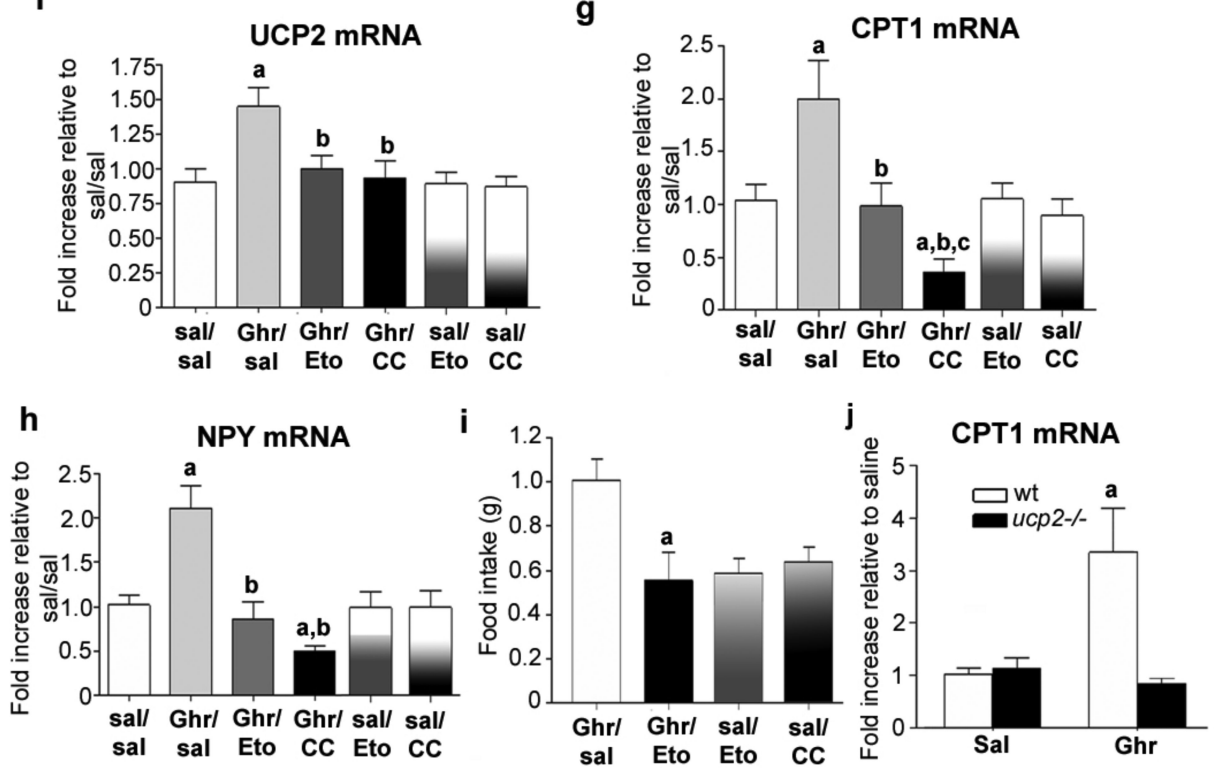

Figure 3. Ghrelin activates an intracellular pathway of fatty acid metabolsim

a, Ghrelin increases the pAMPK/AMPK ratio in wild-type but not $\mathrm{Ghs}^{-1-}$ mice $(n=5)$. Ghr, ghrelin; Sal, saline. b, Ghrelin increases the pAMKP/AMPK ratio and phosphorylates downstream ACC in both wild-type and $U c p 2^{-/-}$mice, indicating that activation of AMPK is upstream to increased UCP2 activity $(n=6)$. $\mathbf{c}-\mathbf{e}$, AMPK regulation affects food intake. $\mathbf{c}$, AICAR significantly increases food intake relative to saline controls in wild-type and $\mathrm{Ghsr}^{-1-}$ mice. d, AICAR-induced food intake is significantly suppressed in $\mathrm{Ucp}^{-{ }^{--}}$mice compared to controls, indicating that UCP2 is required to translate AMPK activity into a 
complete food intake response $(n=5)$. e, AICAR significantly increases cumulative 3-h food intake relative to saline controls; however, this effect is completely blocked with a concurrent intracerebroventricular injection of compound $\mathrm{C}\left(\mathrm{CC}, 6 \mu \mathrm{g} \mu \mathrm{l}^{-1}\right)$, an inhibitor of AMPK activity. AICAR did not affect the cumulative 3-h food intake in $U c p 2^{-/-}$mice and this was not affected by concurrent intracerebroventricular injection of compound C. $\mathbf{f}$, Intraperitoneal ghrelin administration increases hypothalamic Ucp 2 mRNA; however, this increase is blocked by concurrent injection of etomoxir or $\mathrm{CC}$, indicating that activation of the AMPK-CPT1 pathway is important in the regulation of Ucp 2 mRNA expression. $\mathbf{g}$, Intraperitoneal ghrelin administration increases hypothalamic Cpt 1 mRNA expression;

[Author: On the figure, $\mathrm{f}$ is labelled $U c p 3$ mRNA and $\mathrm{g}$ is labelled Cpt1 mRNA. Please correct as necessary.] however, the induction of $C p t 1 \mathrm{mRNA}$ with ghrelin (Ghr) is blocked when the CPT1 inhibitor etomoxir (Eto), or compound C (CC), is injected

intracerebroventricularly. $\mathbf{h}$, Peripheral ghrelin injection increases hypothalamic expression of Npy mRNA, which is completely suppressed by intracerebroventricular injection of etomoxir and CC. Note that in $\mathbf{f}-\mathbf{h}$ mRNA expression was not affected by intracerebroventricular injection of etomoxir or compound $\mathrm{C}$ with saline injected intraperitoneally. [Author: Revised sentence ok?Yes.] i, Inhibition of CPT1 activity with an intracerebroventricular injection of etomoxir suppresses ghrelin-induced (IP $10 \mathrm{nmol}$ ) food intake compared to mice receiving intraperitoneal ghrelin and intracerebroventricular saline (Ghr/Sal). Neither intracerebroventricular etomoxir nor compound $\mathrm{C}$ affected food intake in mice treated intraperitoneally with saline $(n=5-7)$. $\mathbf{j}$, Ghrelin increased Cpt1 mRNA expression in wild-type but not in $U c p 2^{-1-}$ mice $(n=6, P<0.05)$. Asterisk, statistically significant $(P<0.05)$ with respect to saline controls; dagger, statistically significant $(P<0.05)$ with respect to Ghr/Sal. [Author: Please clarify ' $\mathrm{c}$ ' label in panel g; statistically significant $(\mathrm{P}<0.05)$ with respect to $\mathrm{Ghr} /$ Eto.] All error bars indicate s.e.m. 


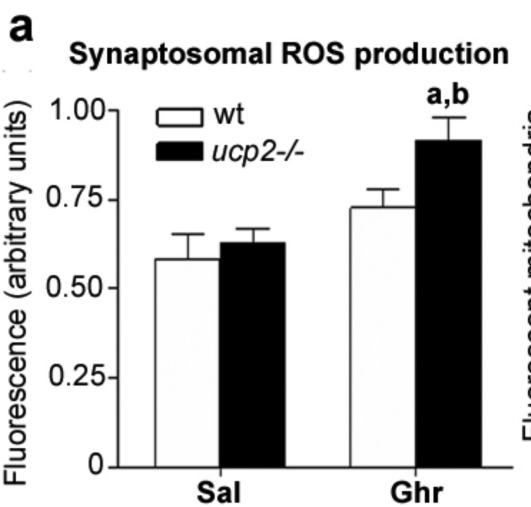

b $\quad$ DHE

C CPT1 mRNA

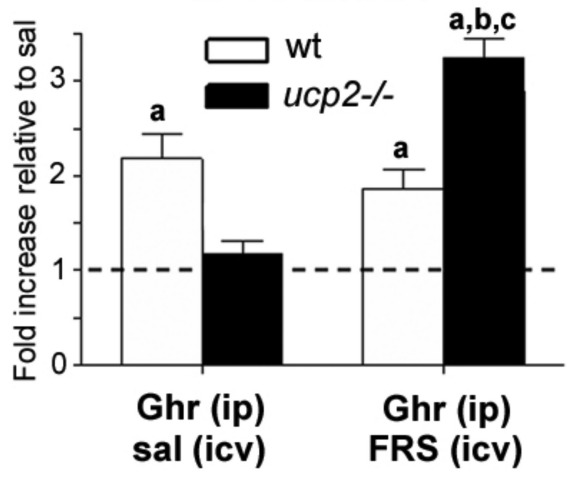

e

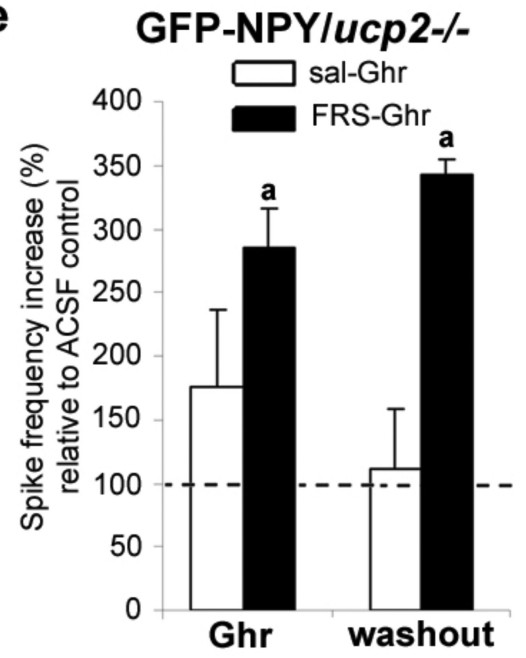

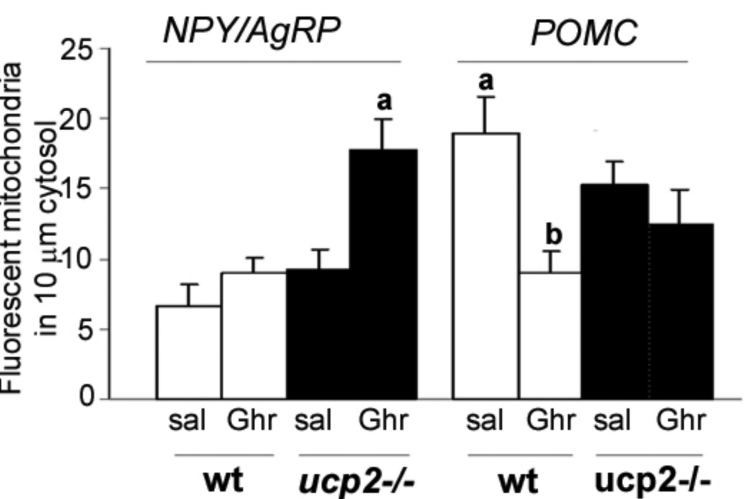

d

NPY mRNA

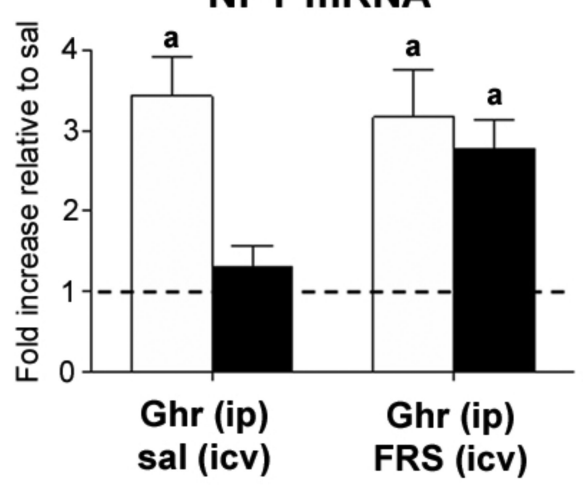

f

Food intake

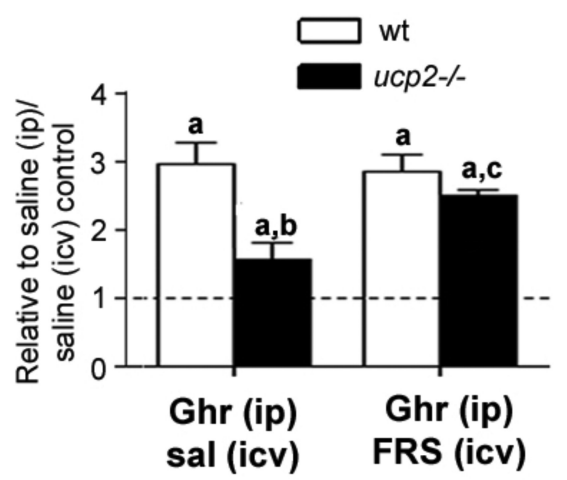

Figure 4. ROS is a critical regulator of cellular and behavioural responses to ghrelina Ghrelin increases hypothalamic synaptosomal ROS production in $U c p 2^{-/-}$mice but not wild-type mice $(n=4-5)$. b, Quantification of ROS production shows that ghrelin markedly increases in situ ROS in NPY neurons in $U c p 2^{-/-}$but not wild-type mice. c, Suppressed Cpt 1 mRNA expression in ghrelin-treated $U c p 2^{-/-}$mice after $3 \mathrm{~h}$ is completely reversed and elevated above wild-type levels by concurrent intracerebroventricular injection of free radical scavenging (FRS) cocktail $(n=5, P<0.05)$. i.c.v., intracerebroventricular; i.p., intraperitoneal. d, FRS cocktail reverses suppressed Npy mRNA in ghrelin-treated $U c p 2^{-/-}$ 
mice. e, Incubating arcuate slices with FRS restores suppressed NPY neuronal action potential firing in $U c p 2^{-/-}$mice. Neurons were from NPY-GFP/Ucp $2^{-/-}$mice. Asterisk, significant to $100 \%$ saline controls. f, The FRS cocktail reverses the suppressed ghrelininduced food intake after $1 \mathrm{~h}$ in $U c p 2^{-l-}$ mice $(n=9, P<0.05)$. Except for e: asterisk, significant to saline controls; dagger, significant decrease compared to wild-type mice administered ghrelin intraperitoneally and saline intracerebroventricularly; double dagger, significant with respect to $U c p 2^{-/-}$mice administered ghrelin intraperitoneally and saline intracerebroventricularly. 